\title{
NUMERICAL SIMULATION OF QUASI-STATIC AND DYNAMIC EXPERIMENTS OF STANDARD AND DAM CONCRETE
}

\author{
F. BOUSIKHANE* ${ }^{*}$ R. REZAKHANI ${ }^{*}$, G. DI LUZIO ${ }^{* *}$, J. SMITH ${ }^{* * *}$, G. CUSATIS ${ }^{*}$ \\ *Northwestern University \\ Evanston, IL USA \\ ** Politecnico di Milano \\ Milano, Italy \\ *** University of the West Indies \\ St. Augustine, Trinidad and Tobago \\ e-mail: faysalbousikhane2014@u.northwestern.edu - roozbehrezakhani2011@u.northwestern.edu - \\ diluzio@stru.polimi.it - jovanca.smith@sta.uwi.edu - g-cusatis@northwestern.edu
}

Key words: Concrete, Aggregate size effect, Quasi-Static, Dynamic, Lattice Discrete Model

\begin{abstract}
Aggregate size effect is among several important factors that affect concrete mechanical behavior. In this study, this effect is investigated numerically, and the obtained results are compared with the gathered experimental data that are recently performed at Politecnico di Milano and the Joint Research Center of Ispra, Italy. Since concrete is a rate-dependent material, different types of static and dynamic experiments are carried out to study the aggregate size effect on concrete response. The Lattice Discrete Particle Model (LDPM), a three-dimensional mesoscale discrete model, is employed to simulate concrete mechanical response. LDPM simulates concrete at the level of coarse aggregate pieces and is capable of characterizing strain localization, distributed cracking in tension and compression and to reproduce post peak softening behavior. The parameters governing different aspects of LDPM from concrete mixture design to the meso-scale mechanical constitutive law are calibrated and used in the validation process.
\end{abstract}

\section{Introduction}

Concrete is the most widely used material in the construction of civil engineering structures such as dams, bridges and buildings. Concrete has a complex heterogeneous internal structure, which includes several characteristic lengths from the scale of cement nano particles to the macroscale. Concrete mechanical properties evolve during its lifetime due to different phenomena such as hydration, creep, shrinkage, drying and alkali-silica-reaction. Besides, concrete is a rate dependent material, which means that its mechanical characteristics, including compressive and tensile strength, young's modulus and fracture energy, vary with respect to the applied loading rate [1,2]. Therefore, con- sidering all aforementioned complexities, a detailed computational model should be developed to accurately simulate concrete behavior.

Computational models that have been developed to simulate concrete cracking and failure can be categorized as cohesive crack models [3], smeared crack approach [4], and discrete lattice and particle models [5], that are all discussed and compared in [6]. In the cohesive crack model, it is assumed that cracking occurs between the boundary of finite elements when the nodal force normal to the element boundary reaches a critical value [7]. That node is then duplicated and separated by the crack, and new degrees of freedom are added to the system. In smeared crack approaches, cracking and 


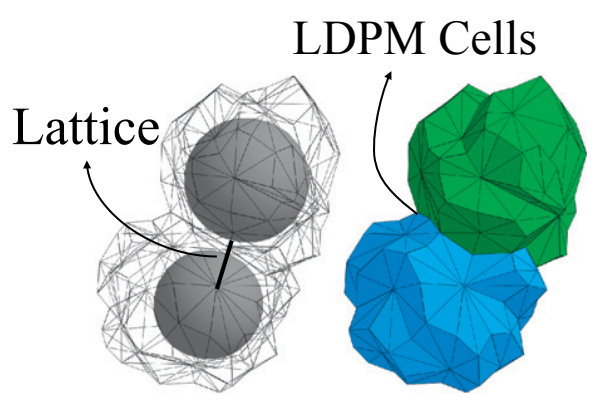

(a)

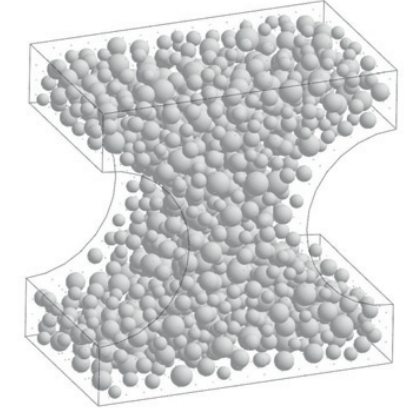

(b)

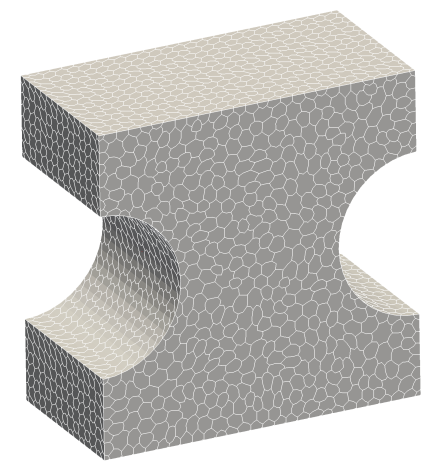

(c)

Figure 1: (a) LDPM polyhedral cell enclosing spherical aggregate pieces. (b) Spherical particle and (c) polyhedral cell representations of a typical dogbone specimen.

damage is assumed to occur within a band of finite elements. Mesh sensitivity of the response is the main drawback of this approach. Discrete models, on the other hand, can simulate material fracture and failure without suffering from the typical drawbacks of continuum based models. In these methods, material domain is approximated a priori by a discrete system of lattices and/or particles. Therefore, their kinematics naturally handle displacement discontinuities and cracking [8]. Among other advantages of discrete models, remeshing during the analysis is not necessary, and the constitutive equations are vectorial as opposed to tensorial. The latter is a key characteristic for the simulation of fracture.

The Lattice Discrete Particle Model (LDPM), recently developed by Cusatis et al. [9, 10] is a meso-scale model which simulates concrete at the scale of coarse aggregate pieces. LDPM has successfully captured concrete fracture and failure in multiple types of experiments and has been extended to model ASR effect [11-13], fiber reinforced concrete [14], fracture and failure of concrete in a multiscale framework [15, 16], and simulating rock mechanics under different loading conditions [17]. In the current research, LDPM is calibrated and validated with respect to the experimental data recently provided on several quasi-static and dynamic experiments performed at Politecnico di Milano and the Joint Research Center of
Ispra, respectively. These experiments were carried out on two different types of concrete: standard and dam concrete which vary in their mixture design and mainly on their maximum aggregate size. Furthermore, different sizes of specimens are considered for each test to investigate the specimen size effect in addition to aggregate size effect. LDPM parameters relevant to quasi-static and dynamic experiments are calibrated by methodically fitting the results of the numerical simulations with the provided experimental data. Using the calibrated parameters, the validation procedure is performed by simulating the experiments that that were not used in the calibration process.

\section{The Lattice Discrete Particle Model (LDPM)}

LDPM constructs the geometrical representation of concrete meso-structure through the following steps. 1) The coarse aggregate pieces, whose shapes are assumed to be spherical, are introduced into the concrete volume by a try-and-reject random procedure. 2) Zeroradius aggregate pieces (nodes) are randomly distributed over the external surfaces to facilitate the application of boundary conditions. 3) A three-dimensional domain tessellation, based on the Delaunay tetrahedralization of the generated aggregate centers, creates a system of polyhedral cells (see Figure 1a) interacting through triangular facets and a lattice system composed 


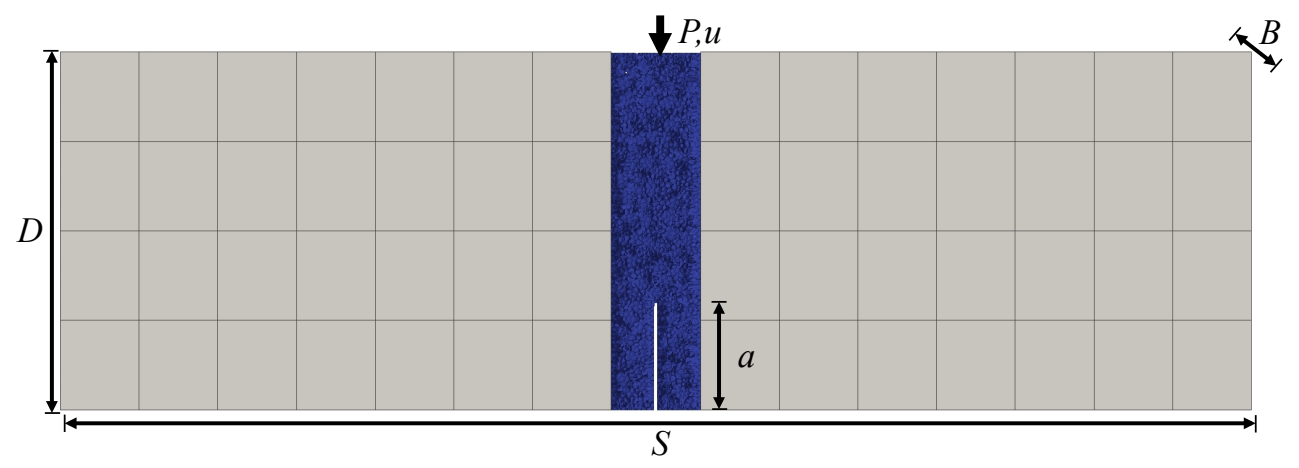

Figure 2: Geometry of three-point bending test specimens

\begin{tabular}{|c||c|c|c|c|}
\hline Dam Concrete Specimen & Span $S[\mathrm{~mm}]$ & Height $D[\mathrm{~mm}]$ & Width $B[\mathrm{~mm}]$ & Notch depth $a[\mathrm{~mm}]$ \\
\hline Small & 900 & 300 & 240 & 90 \\
Medium & 1800 & 600 & 240 & 180 \\
Large & 2700 & 900 & 250 & 270 \\
\hline Standard Concrete Specimen & Span $S[\mathrm{~mm}]$ & Height $D[\mathrm{~mm}]$ & Width $B[\mathrm{~mm}]$ & Notch depth $a[\mathrm{~mm}]$ \\
\hline Small & 450 & 150 & 120 & 45 \\
Medium & 900 & 300 & 120 & 90 \\
Large & 1800 & 600 & 120 & 180 \\
\hline
\end{tabular}

Table 1: Three point bending specimen dimensions for dam and standard concrete.

by the line segments connecting the particle centers. Figures 1 c and d represent spherical particle and corresponding polyhedral representations of a typical dogbone specimen.

In LDPM, rigid body kinematics is used to describe the deformation of the lattice/particle system and the displacement jump, $\llbracket \mathbf{u}_{C} \rrbracket$, at the centroid of each facet is used to define measures of strain as

$e_{N}=\frac{\mathbf{n}^{\mathrm{T}} \llbracket \mathbf{u}_{C} \rrbracket}{\ell} ; \quad e_{L}=\frac{\mathbf{l}^{\mathrm{T}} \llbracket \mathbf{u}_{C} \rrbracket}{\ell} ; \quad e_{M}=\frac{\mathbf{m}^{\mathrm{T}} \llbracket \mathbf{u}_{C} \rrbracket}{\ell}$

where $\ell=$ interparticle distance; and $\mathbf{n}, \mathbf{l}$, and $\mathbf{m}$, are unit vectors defining a local system of reference attached to each facet.

Next, a vectorial constitutive law governing the behavior of the material is imposed at the centroid of each facet. In the elastic regime, the normal and shear stresses are proportional to the corresponding strains: $t_{N}=E_{N} e_{N}^{*}=$ $E_{N}\left(e_{N}-e_{N}^{0}\right) ; t_{M}=E_{T} e_{M}^{*}=E_{T}\left(e_{M}-e_{M}^{0}\right) ; t_{L}=$ $E_{T} e_{L}^{*}=E_{T}\left(e_{L}-e_{L}^{0}\right)$, where $E_{N}=E_{0}, E_{T}=\alpha E_{0}$, $E_{0}=$ effective normal modulus, and $\alpha=$ shearnormal coupling parameter; and $e_{N}^{0}, e_{M}^{0}, e_{L}^{0}$ are mesoscale eigenstrains that might arise from a variety of phenomena such as, but not limited to, thermal expansion, shrinkage, and ASR expansion.

For stresses and strains beyond the elastic limit, LDPM mesoscale nonlinear phenomena are characterized by three mechanisms as described below.

Fracture and cohesion due to tension and tension-shear. For tensile loading $\left(e_{N}^{*}>0\right)$, the fracturing behavior is formulated through an effective strain, $e=\sqrt{e_{N}^{* 2}+\alpha\left(e_{M}^{* 2}+e_{L}^{* 2}\right)}$, and stress, $t=\sqrt{t_{N}^{2}+\left(t_{M}+t_{L}\right)^{2} / \alpha}$, which define the normal and shear stresses as $t_{N}=e_{N}^{*}(t / e) ; \quad t_{M}=\alpha e_{M}^{*}(t / e) ; \quad t_{L}=\alpha e_{L}^{*}(t / e)$. The effective stress $t$ is incrementally elastic $\left(\dot{t}=E_{0} \dot{e}\right)$ and must satisfy the inequality $0 \leq t \leq \sigma_{b t}(e, \omega)$ where $\sigma_{b t}=$ $\sigma_{0}(\omega) \exp \left[-H_{0}(\omega)\left\langle e-e_{0}(\omega)\right\rangle / \sigma_{0}(\omega)\right],\langle x\rangle=$ $\max \{x, 0\}$, and $\tan (\omega)=e_{N}^{*} / \sqrt{\alpha} e_{T}^{*}=t_{N} \sqrt{\alpha} / t_{T}$, $e_{T}^{*}=\sqrt{e_{M}^{* 2}+e_{L}^{* 2}}$, and $e_{0}(\omega)=\sigma_{0}(\omega) / E_{0}$. The 


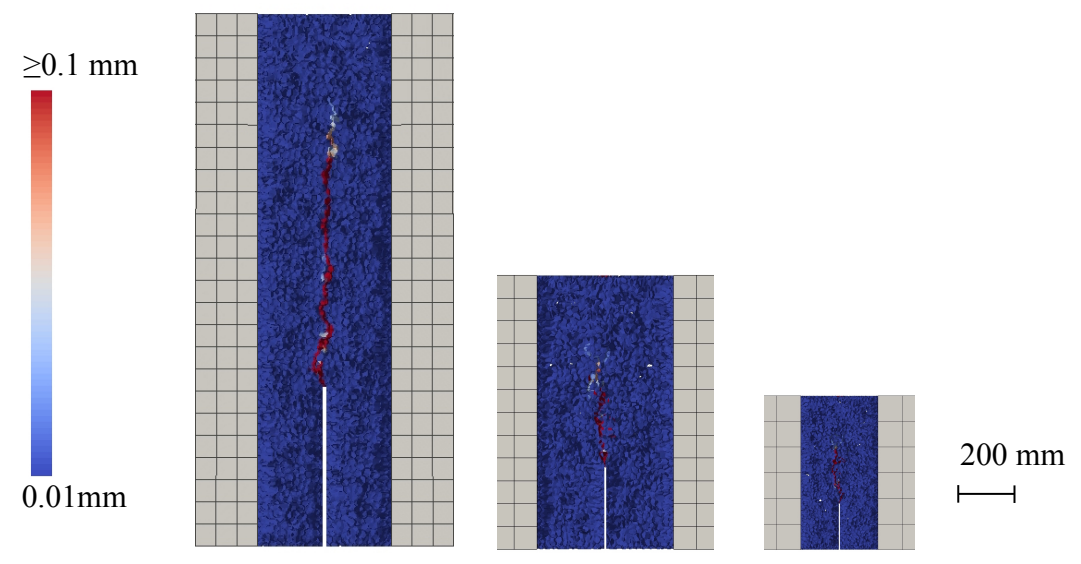

Figure 3: Crack opening contour of dam concrete three point bending specimens of different sizes at failure.

post peak softening modulus is defined as $H_{0}(\omega)=H_{t}(2 \omega / \pi)^{n_{t}}$, where $H_{t}$ is the softening modulus in pure tension $(\omega=\pi / 2)$ expressed as $H_{t}=2 E_{0} /\left(l_{t} / l_{e}-1\right) ; l_{t}=2 E_{0} G_{t} / \sigma_{t}^{2} ; l_{e}$ is the length of the tetrahedron edge; and $G_{t}$ is the mesoscale fracture energy. LDPM provides a smooth transition between pure tension and pure shear $(\omega=0)$ with parabolic variation for strength given by $\sigma_{0}(\omega)=\sigma_{t} r_{s t}^{2}(-\sin (\omega)$ $\left.+\sqrt{\sin ^{2}(\omega)+4 \alpha \cos ^{2}(\omega) / r_{s t}^{2}}\right) /\left[2 \alpha \cos ^{2}(\omega)\right]$, where $r_{s t}=\sigma_{s} / \sigma_{t}$ is the ratio of shear strength to tensile strength.

\section{Compaction and pore collapse from com-} pression. Normal stresses for compressive loading $\left(e_{N}^{*}<0\right)$ are computed through the inequality $-\sigma_{b c}\left(e_{D}^{*}, e_{V}^{*}\right) \leq t_{N} \leq 0$, where $\sigma_{b c}$ is a strain-dependent boundary function of the volumetric strain, $e_{V}^{*}$, and the deviatoric strain, $e_{D}^{*}=e_{N}^{*}-e_{V}^{*}$. The volumetric strain is computed by the volume variation of the Delaunay tetrahedra as $e_{V}^{*}=\Delta V / 3 V_{0}$ and is assumed to be constant for all facets belonging to a given tetrahedron. Beyond the elastic limit, $-\sigma_{b c}$ models pore collapse as a linear evolution of stress for increasing volumetric strain with stiffness $H_{c}$ for $-e_{V}^{*} \leq e_{c 1}^{*}=\kappa_{c 0} e_{c 0}^{*}$ : $\sigma_{b c}=\sigma_{c 0}+\left\langle-e_{V}^{*}-e_{c 0}^{*}\right\rangle H_{c}\left(r_{D V}\right) ; H_{c}=\left(H_{c 0}+\right.$ $\left.H_{c 1}\right) /\left(1+\kappa_{c 2}\left\langle r_{D V}-\kappa_{c 1}\right\rangle\right)+H_{c 1}$ with $\left.H_{c 1}\right\rangle$ $H_{c} 0 ; \sigma_{c 0}$ is the mesoscale compressive yield stress; $r_{D V}=\left|e_{D}\right| /\left(e_{V}^{*}-e_{r 0}\right)$ for $e_{V}^{*} \leq 0$ and
$r_{D V}=\left|e_{D}^{*}\right| / e_{V 0}$ for $e_{V}^{*}>0, e_{V O}^{*}=k_{c 3} \epsilon_{0}, k_{c 3}=$ 0.1 and $\kappa_{c 1}, \kappa_{c 2}$ are material parameters. Compaction and rehardening occur beyond pore collapse $\left(-e_{V}^{*} \geq e_{c 1}^{*}\right)$. In this case one has $\sigma_{b c}=$ $\sigma_{c 1}\left(r_{D V}\right) \exp \left[\left(-e_{V}^{*}-e_{c 1}^{*}\right) H_{c}\left(r_{D V}\right) / \sigma_{c 1}\left(r_{D V}\right)\right]$ and $\sigma_{c 1}\left(r_{D V}\right)=\sigma_{c 0}+\left(e_{c 1}^{*}-e_{c 0}^{*}\right) H_{c}\left(r_{D V}\right)$.

Friction due to compression-shear. The incremental shear stresses are computed as $\dot{t}_{M}=E_{T}\left(\dot{e}_{M}^{*}-\dot{e}_{M}^{* p}\right)$ and $\dot{t}_{L}=E_{T}\left(\dot{e}_{L}^{*}-\dot{e}_{L}^{* p}\right)$, where $\dot{e}_{M}^{* p}=\dot{\lambda} \partial \varphi / \partial t_{M}, \dot{e}_{L}^{* p}=\dot{\lambda} \partial \varphi / \partial t_{L}$, and $\lambda$ is the plastic multiplier with loading-unloading conditions $\varphi \dot{\lambda} \leq 0$ and $\dot{\lambda} \geq 0$. The plastic potential is defined as $\varphi=\sqrt{t_{M}^{2}+t_{L}^{2}}-\sigma_{b s}\left(t_{N}\right)$, where the nonlinear frictional law for the shear strength is assumed to be $\sigma_{b s}=\sigma_{s}+\left(\mu_{0}-\mu_{\infty}\right) \sigma_{N 0}[1-$ $\left.\exp \left(t_{N} / \sigma_{N 0}\right)\right]-\mu_{\infty} t_{N} ; \sigma_{N 0}$ is the transitional normal stress; $\mu_{0}$ and $\mu_{\infty}$ are the initial and final internal friction coefficients.

Finally, the governing equations of the LDPM framework are completed through the equilibrium equations of each individual particle.

Rate dependence of LDPM is captured by introducing a rate dependent function into the facet strain dependent boundary function previously described in Section 2 as $\sigma_{b t}=$ $F(\dot{\omega}) \sigma_{0}(\omega) \exp \left[-H_{0}(\omega)\left\langle e-e_{0}(\omega)\right\rangle / \sigma_{0}(\omega)\right]$ where rate dependent function $F(\dot{\omega})=1+$ $c_{1} \operatorname{asinh}\left(\dot{e} /\left(c_{0} / l\right)\right)$, see [18] for details. 
(a)
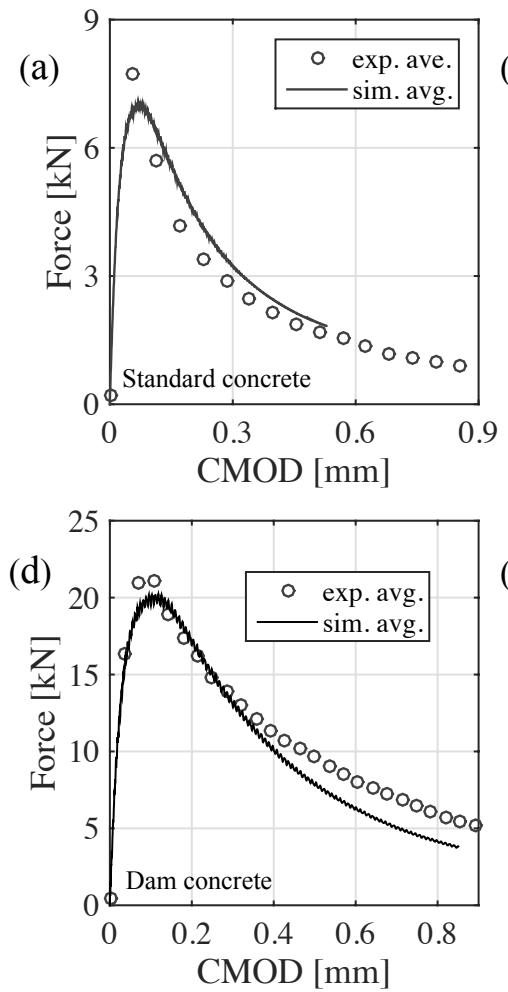

(b)
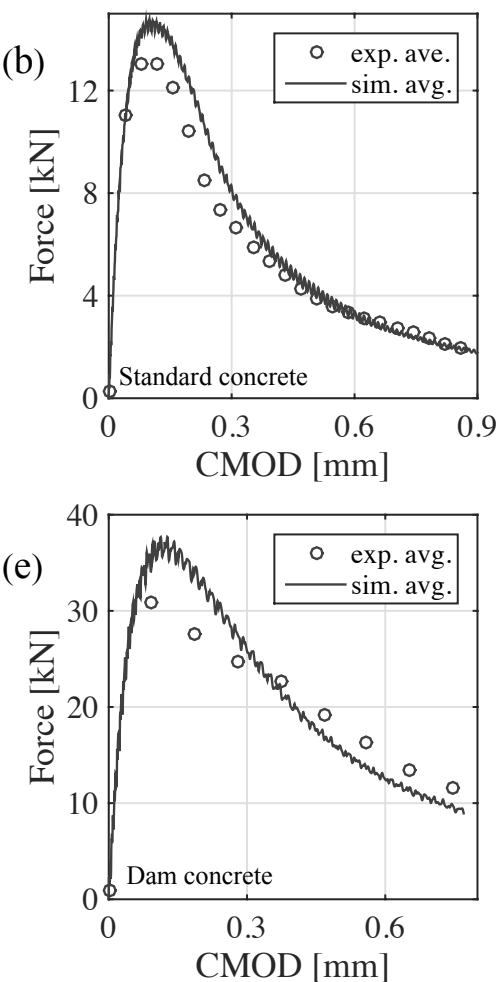
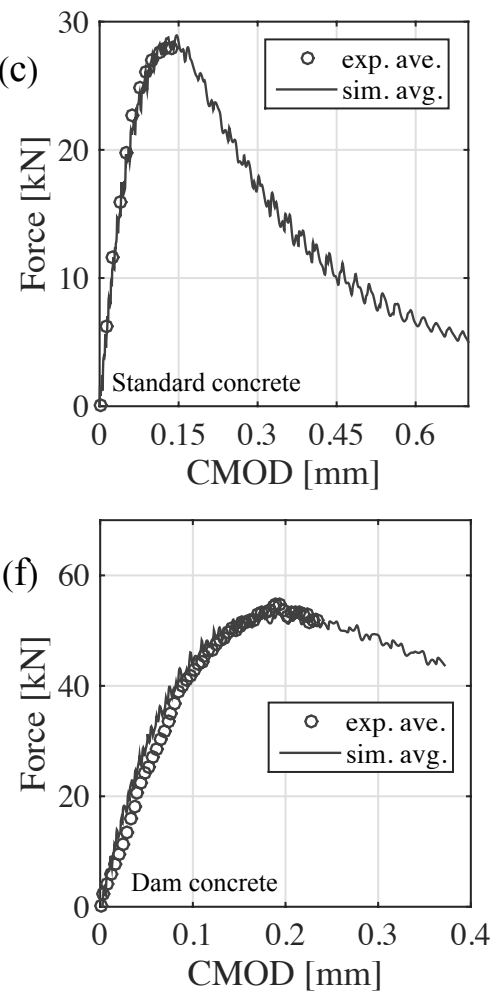

Figure 4: Force versus CMOD curves for small, medium, and large specimens for (a) to (c) standard and (d) to (f) dam concrete.

\section{Calibration and validation of LDPM}

LDPM parameters that need to be defined split into two primary groups: The first set characterizes the geometry and composition of concrete meso-structures. It includes water to cement ratio $w / c$, aggregate to cement ratio $a / c$, the maximum $d_{a}$ and minimum $d_{0}$ aggregate size, and the Fuller coefficient $n_{f}$ which is used to obtain the aggregate size distribution [9]. The Fuller coefficient value was adjusted by fitting the theoretical fuller curve formula to the sieve curve of the standard and dam concrete used in the experiments. All other parameters were based on the mix designs that are provided for both standard and dam concrete. Mix design parameters for dam concrete were: cement content $c=236 \mathrm{~kg} / \mathrm{m}^{3}, a / c=8.82, w / c=0.5, d_{0}$ $=10 \mathrm{~mm}, d_{a}=64 \mathrm{~mm}$, and $n_{f}=0.5$. For standard concrete they were $c=273 \mathrm{~kg} / \mathrm{m}^{3}, a / c=$ 7.33, $w / c=0.55, d_{0}=6 \mathrm{~mm}, d_{a}=30 \mathrm{~mm}$, and $n_{f}=0.5$. The second set of LDPM param- eters are related to the meso-scale mechanical behavior which is formulated by the constitutive equations explained in Section 2, Different subsets of this set of parameters are calibrated with respect to the results of different types of performed experiments.

\subsection{Static Simulations}

Simulation of quasi-static experiments including three point bending, uniaxial unconfined compression, and confined compression are considered in this section. In all simulations, three LDPM particle realizations are considered, and the averaged results are plotted for each test.

\subsubsection{Three Point Bending}

Three point bending experiments are carried out on three different specimen sizes for dam and standard concrete which are reported in Figure 2 and Table 2. One can see that the 

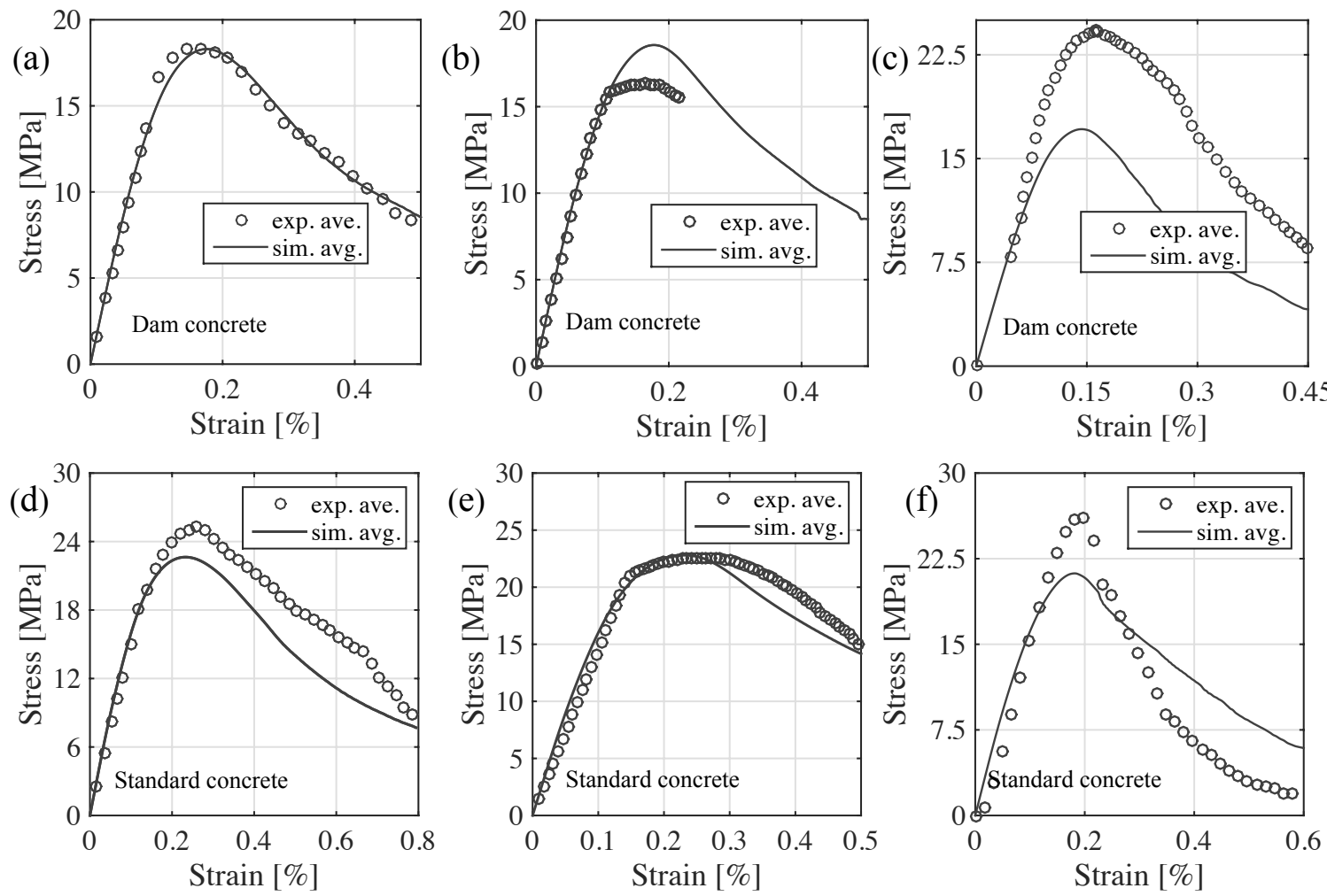

Figure 5: Stress-Strain curves of unconfined compression tests for (a) and (b) small and large dam concrete cylinders, (c) dam concrete prism, (d) and (e) small and large standard concrete cylinders, (f) standard concrete prism.

dam concrete samples are larger than standard ones due to the larger maximum aggregate size. Standard clip gauges were used to measure the Crack Mouth Opening Displacement (CMOD) during the loading process. The softening behavior due to tensile fracturing are governed by tensile strength $\sigma_{t}$ and meso-scale characteristic length $l_{t}$ (related to the fracture energy $G_{t}$ through $\left.l_{t}=2 E_{0} G_{t} / \sigma_{t}^{2}\right)$ that are calibrated using the three point bending test results. To save the computational cost, only the middle part of the specimens are simulated by LDPM, which is the volume where that fracture localization is expected to occur. The two sides of the specimen are modeled by elastic finite element. Force versus CMOD curves for the standard and dam concrete of three different sizes specimens are presented in Figure 4. LDPM parameters calibration procedure was performed through fitting the load CMOD curve of the small speci- mens with the experimental data, and the model is validated by predicting the results for the two other sizes. In the case of standard concrete, the large, medium, and small specimens show respectively an average peak load around $29 \mathrm{kN}$, $13 \mathrm{kN}$ and $7 \mathrm{kN}$, while for dam concrete are $55 \mathrm{kN}, 37 \mathrm{kN}$ and $24 \mathrm{kN}$. It is shown that the experimental data and the numerical simulation results are in excellent agreement for all different sizes with respect to peak loads and post peak softening behavior, which also demonstrates LDPM capability to characterize size effect. However, in the case of small and medium dam concrete specimens, post peak softening behavior is less accurate. This can be explained by the fact that the maximum aggregate size is relatively large, hence the number of aggregates that can fit along the ligament above the notch is relatively small, which leads to a lack of resolution of the crack pattern. 


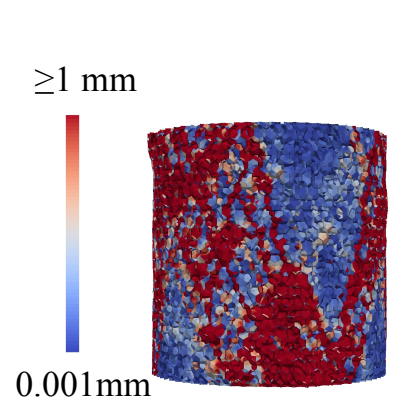

(a)

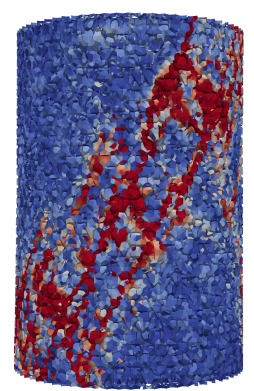

(b)

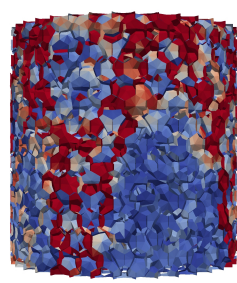

(c)

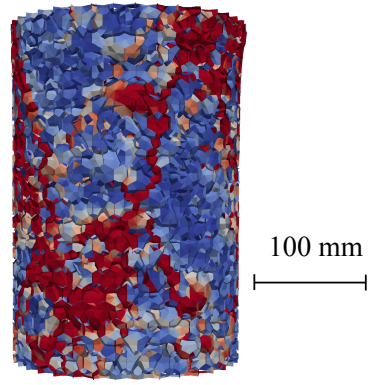

(d)

Figure 6: Contours of meso-scale crack opening at failure for (a) small and (b) large standard and (c) small and (d) large dam cylindrical specimens.
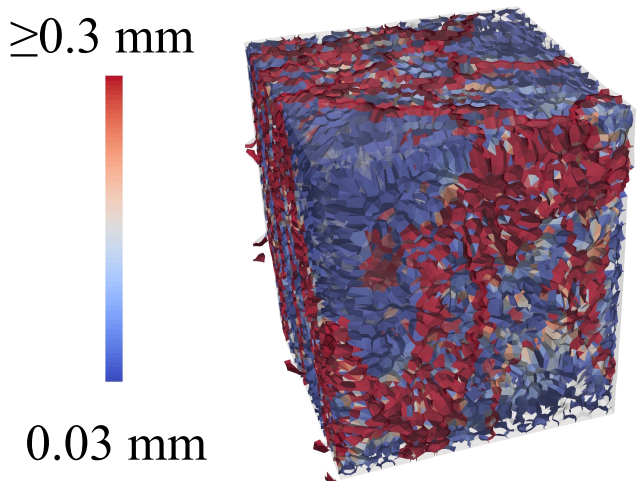

(a)

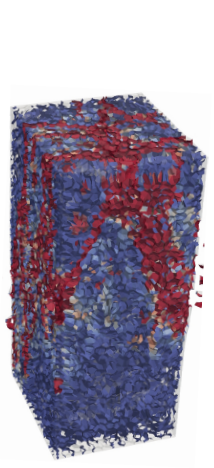

(b)

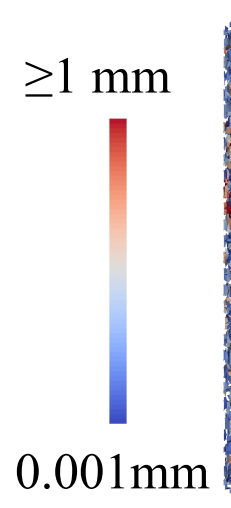

(1.

(c)

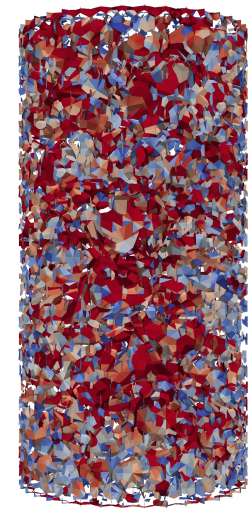

(d)

Figure 7: Contours of meso-scale crack opening at failure for uniaxial compression of (a) dam and (b) standard prisms. Meso-scale crack opening contour of confined compression simulations at failure for standard concrete with a (a) $4 \mathrm{~mm}$ and (b) $10 \mathrm{~mm}$ thickness jackets.

\subsubsection{Unconfined Compression}

In unconfined compression test, LDPM parameters that need to be calibrated are the shear strength $\sigma_{s}$ and the initial friction $\mu_{0}$ which affect mostly the peak value, the softening exponent $n_{t}$ that governs the post peak behavior. Prismatic $(240 \mathrm{~mm} \times 240 \mathrm{~mm} \times 300 \mathrm{~mm}$ for dam and $117 \mathrm{~mm} \times 137 \mathrm{~mm} \times 300 \mathrm{~mm}$ for standard concrete) and cylindrical specimens (200 $\mathrm{mm}$ and $300 \mathrm{~mm}$ lengths and $200 \mathrm{~mm}$ diameter for both concrete types) are used in the unconfined compression experiments. As shown in Figure 5, stress-strain curves of the numerical simulations are in good agreement with the experimental data. One can observe that the experimental peak values obtained for different types of standard concrete specimens are con- sistent, while there is a discrepancy between the peak values obtained for the dam concrete prismatic specimen and the cylindrical ones. This can be justified by the low ratio of the specimen size to the maximum aggregate size, and the fact that only one sample was tested for the dam concrete prismatic case. As shown in Figures 6 and 7 (a) and (b), LDPM replicates the shear mode failure with oblique crack patterns which is consistent with what is observed in experiments.

\subsubsection{Confined Compression}

In the confined compression tests the specimens are axially loaded inducing a lateral deformation which is confined by a steel jacket generating an inward reaction force setting the 

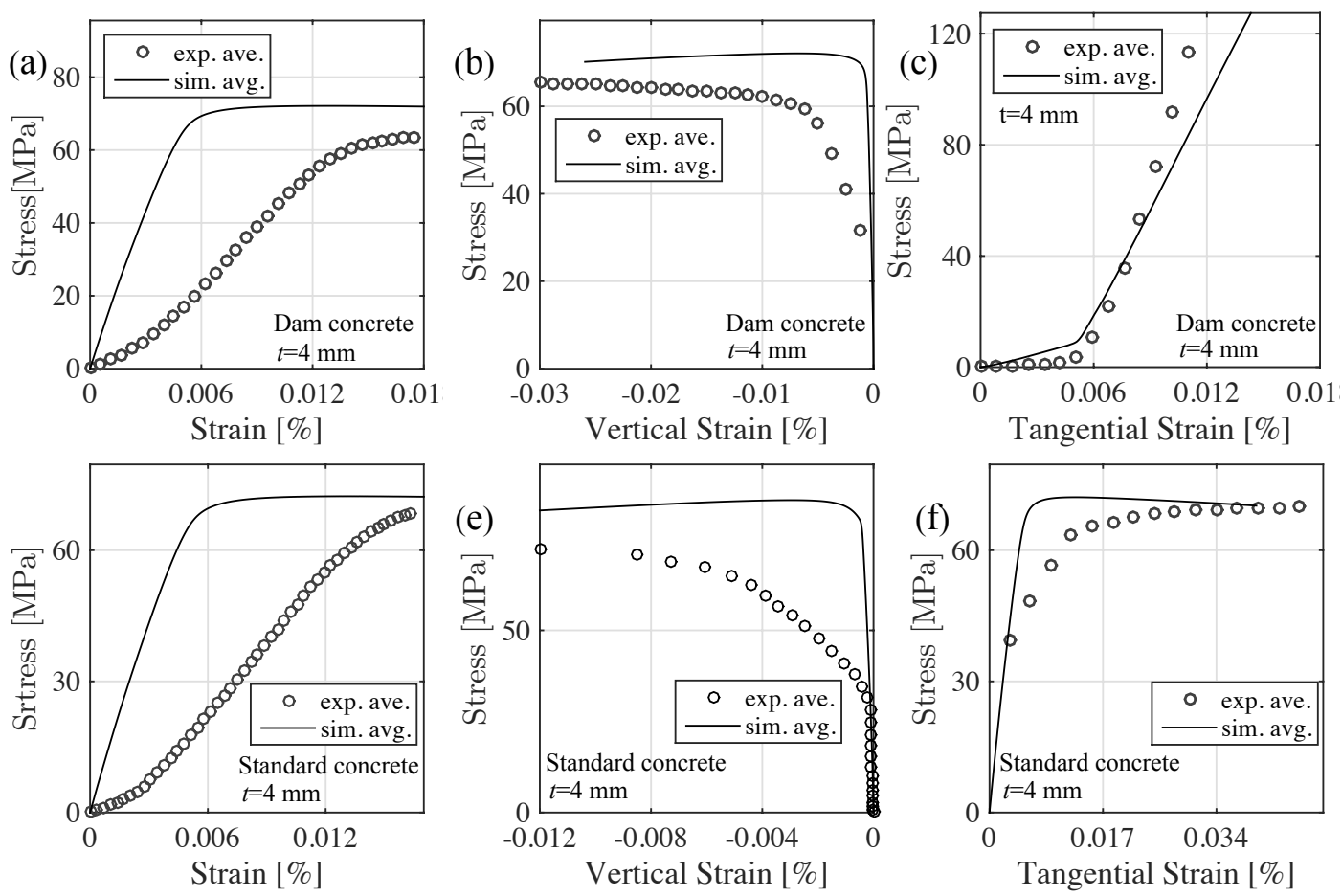

Figure 8: Confined compression tests using $4 \mathrm{~mm}$ thickness jacket. Force versus total strain, ,vertical strain and tangential strain curves for dam respectively (a), (b), (c) and standard concrete respectively (d), (e), (f) cylinders.

specimen under a state of triaxial confined pressure. Cylinders of $200 \mathrm{~mm}$ diameter and 400 mm height confined by steel jackets of 4 and $10 \mathrm{~mm}$ thickness are used in the experiments. The instrumentation used for data acquisition were two strain-gauges and one LVDT. The two strain-gauges were placed at the mid-height of the steel jacket to measure the tangential and vertical strains, and one that measures the total longitudinal deformation from plate to plate.

The LDPM parameters that must be calibrated are the initial and final hardening modulus ratios $H_{c 0} / E_{0}$ and $H_{c 1} / E_{0}$, respectively. Longitudinal stress versus tangential strain data were used to calibrate the model, while the experimental data for the total vertical strain and LVDT vertical strain served for the validation procedure. Comparison of the numerical simulation results and the experimental data for both concrete types are depicted in Figures 8 and 9 for $4 \mathrm{~mm}$ and $10 \mathrm{~mm}$ steel jacket thickness, respectively. In the stress versus total vertical strain plots, one can see that the numerical results and the experimental data do not match in the initial part for both types of concrete and steel jacket thicknesses. This can be mainly explained by non-uniform initial contact between the loading plates and the specimen ends. This behavior is not observed in Figures 8 (b) and (c) and 9 (b) and (c), since the stain-gauges were directly attached to the jackets. As expected for a constant longitudinal strain, more force is needed in the case of the $10 \mathrm{~mm}$ jacket than the $4 \mathrm{~mm}$ one. As far as aggregate size is concerned, dam concrete shows a bigger capacity to resist triaxial confinement as shown in Figures 8 and 9

The final set of calibrated parameters used for the LDPM simulations of dam concrete are $E_{0}=33340 \mathrm{MPa}, \alpha=0.25, \sigma_{t}=1.55 \mathrm{MPa}$, $\sigma_{s} / \sigma_{t}=1.35, \sigma_{c 0}=150 \mathrm{MPa}, L_{t}=5500 \mathrm{~mm}$, $n_{t}=0.3, H_{c 0} / E_{0}=0.3, \mu_{0}=0.2, k_{c 0}=2, k_{c 1}=$ $1, k_{c 2}=5$, and $H_{c 1} / E_{0}=0.8$. For standard concrete, the final calibrated parameters are normal 

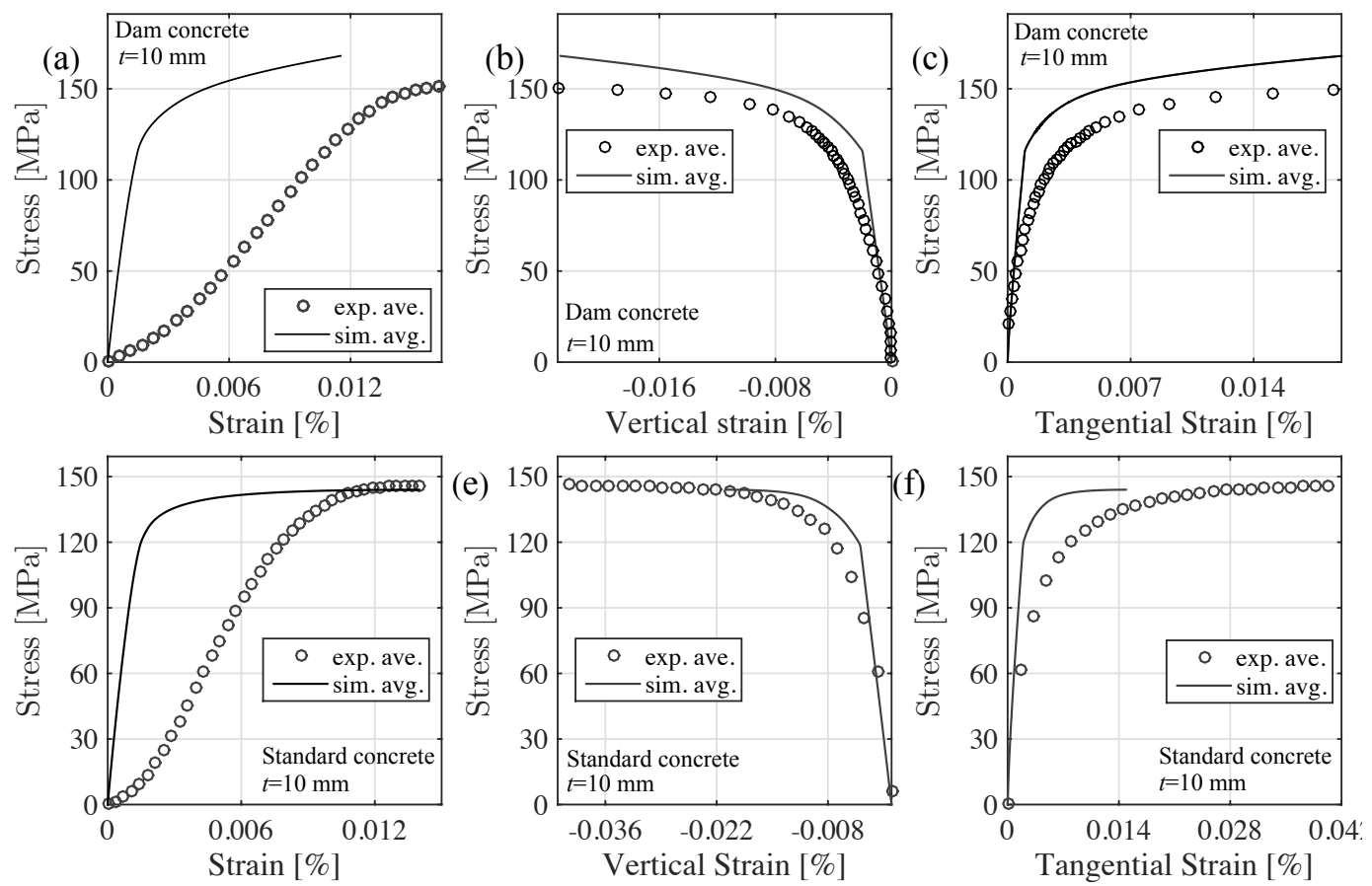

Figure 9: Confined compression tests using $10 \mathrm{~mm}$ thickness jacket. Force versus total strain, ,vertical strain and tangential strain curves for dam respectively (a), (b), (c) and standard concrete respectively (d), (e), (f) cylinders.

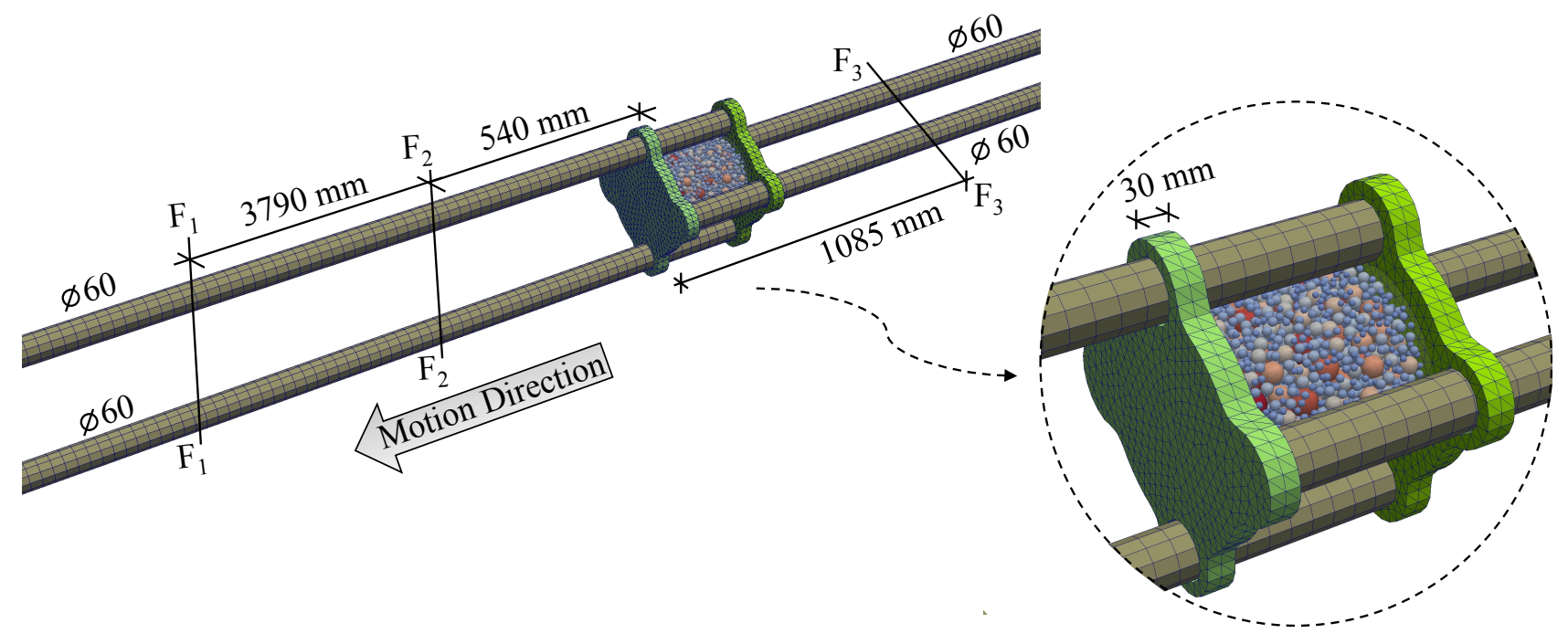

Figure 10: Dynamic compression experiment setup.

modulus $E_{0}=33340 \mathrm{MPa}, \alpha=0.167, \sigma_{t}=2.4$ $\mathrm{MPa}, \sigma_{s} / \sigma_{t}=1.7, \sigma_{c 0}=155 \mathrm{MPa}, L_{t}=2200$ $\mathrm{mm}, n_{t}=0.3, H_{c 0} / E_{0}=0.4$, initial friction $\mu_{0}=$ $0.05, k_{c 0}=2, k_{c 1}=1, k_{c 2}=5$ and $H_{c 1} / E_{0}=0.9$

\subsection{Dynamic Simulations}

Dynamics experiments conducted at the HOPLAB facility on standard and dam concrete specimens are considered in this section to investigate the mechanical characteristics of concrete under high strain-rates. The experimen- 

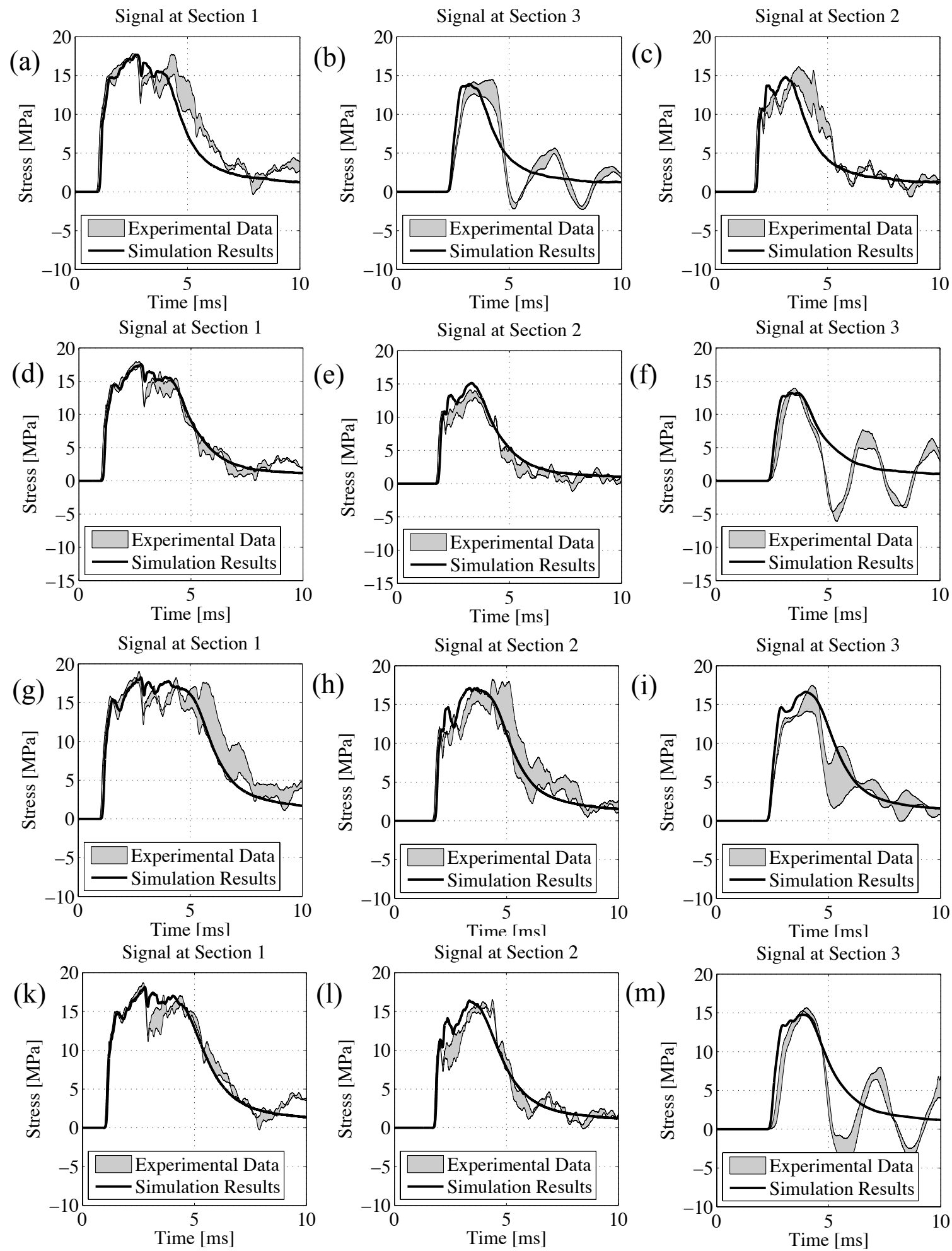

Figure 11: Force measured at sections $F_{1}, F_{2}$, and $F_{3}$ divided by the sample cross section area during dynamic compression tests on (a) to (c) small and (d) to (f) large dam concrete specimens and on (g) to (i) small and (k) to (m) large standard concrete specimens.

tal apparatus which is developed based on the Hopkinson bar techniques is depicted in Figure
10 where the concrete cylindrical specimen is placed between two steel loading plates that are 
each connected to a twin incident and transmitter steel bars. Young modulus and mass density of the steel plates and the twin bars are $E=210$ and $197 \mathrm{GPa}$ and $\rho=7800$ and 7780 $\mathrm{kg} / \mathrm{m}^{3}$, respectively. Sudden breaking bolt at the far left end of the apparatus generates a tensile pulse which travels through the input twin bars. Due to the special developed experimental setup, transferred tensile signal is converted into compressive force and is applied on the specimen by means of the steel loading plates. Part of the compressive wave is transmitted through the concrete specimen and the output twin bars, while part of it is reflected back through the input twin bars. The signal is recorded during the experiment at three different sections shown as $F_{1}, F_{2}$, and $F_{3}$ in Figure 10 . Compression tests are performed on cylindrical specimens of 200 $\mathrm{mm}$ diameter and $200 \mathrm{~mm}$ and $400 \mathrm{~mm}$ lengths for both type of concrete.

For the numerical simulations, the force history that should be applied on the input twin bars is calculated based on the recorded signal at section $\mathrm{F}_{1}$ during the experiment. Longitudinal wave speed is calculated based on the mechanical properties of the twin bars, which is used to approximate the amount of time that takes for the longitudinal wave to travel from the section $F_{1}$ to the specimen and reflects back to the section $F_{1}$. Therefore, one can estimate the initial portion of the signal at the section $F_{1}$ that is not affected by the reflected wave. The estimated signal is used as the loading history in the numerical simulations and is kept as constant to the end of the analysis. Figure 11 shows the comparison between the measured signals at sections $F_{1}, F_{2}$, and $F_{3}$ divided by the sample cross section area from numerical simulation results and the experimental data. It is shown that LDPM can perfectly capture the peak value and the force attenuation trend at three different measurement sections for small and large standard and dam concrete specimens. It should be noted that the signal oscillations observed in experimental data recorded at section $\mathrm{F}_{3}$ is due to machine vibrations that is not captured in numerical simulations.
Parameter $c_{0}$ and $c_{1}$ are calibrated with respect to the small specimens experimental data and are validated through fitting the data for large specimens. $c_{0}=1 \times 10^{-} 6$ and $5 \times 10^{-} 6 / \mathrm{s}$ and $c_{1}=0.12$ and 0.07 are the calibrated values for dam and standard concrete, respectively.

\section{Conclusions}

LDPM calibrated and validated in this paper can accurately simulate different types of quasistatic experiments from fracture to unconfined and confined compression tests. Effect of the specimen size as well as the the aggregate size effect is captured in LDPM simulations. In addition, LDPM can successfully predict concrete behavior under dynamic compressive load in Hopkinson bar experiments, and it can accurately reproduce the force history recorded before and after the specimen in terms of peak values as well as their attenuation trend.

\section{REFERENCES}

[1] Dilger WH, Koch R, Kowalczyk R. Ductility of plain and confined concrete under different strain rates. J Am Concrete Inst 1984;81(1): 73-81.

[2] J. Smith, G. Cusatis, D. Pelessone, E. Landis, J. O'Daniel, J. Baylot. Discrete modeling of ultra-high-performance concrete with application to projectile penetration. International Journal of Impact Engineering 2014; 65: 13-32.

[3] Ngo, D. and Scordelis, A.C. Finite element analysis of reinforced concrete beams, Journal of the American Concrete Institute 1967, 67: 152-163.

[4] Rashid, Y.R. Analysis of prestressed concrete pressure vessels, Nuclear Engineering and Design 1968, 7: 334-344.

[5] Schlangen, E. Experimental and Numerical Analysis of Fracture Processes in Concrete, Dissertation, Delft University of Technology, Delft 1993. 
[6] R. De Borst. Some recent developments in computational modelling of concrete fracture. International Journal of Fracture 1997; 86: 5-36.

[7] M.H. Motamedi, D.A. Weed, C.D. Foster. Numerical simulation of mixed mode (I and II) fracture behavior of pre-cracked rock using the strong discontinuity approach. International Journal of Solids and Structures 2016; 85-86: 44-56.

[8] F. J. Vernerey and M. Kabiri. Adaptive Concurrent Multiscale model for fracture and crack propagation in heterogeneous material. Comput. Methods Appl. Mech. Engrg. 2014; 276: 566-588.

[9] G. Cusatis, D. Pelessone, A. Mencarelli. Lattice Discrete Particle Model (LDPM) for failure behavior of concrete. I: Theory. Cement and Concrete Composites 2011; 33: 881-890.

[10] G. Cusatis, A. Mencarelli, D. Pelessone, J. Baylot. Lattice Discrete Particle Model (LDPM) for failure behavior of concrete. II: Calibration and validation. Cement and Concrete Composites 2011; 33: 891-905.

[11] M. Alnaggar, G. Cusatis, G. Di Luzio. Lattice Discrete Particle Modeling (LDPM) of Alkali Silica Reaction (ASR) deterioration of concrete structures. Cement \& Concrete Composites 2013; 41: 45-59.

[12] G. Cusatis, R. Rezakhani, M. Alnaggar, X. Zhou, and D. Pelessone, Multiscale computational models for the simulation of concrete materials and structures, pages
23-38, CRC Press, 2015/02/26 2014, ISBN 978-1-138-00145-9.

[13] G. Cusatis, M. Alnaggar, R. Rezakhani. Multiscale modeling of Alkali Silica reaction degradation of concrete. CONMOD 2014: 431-438, DOI: 10.13140/2.1.3783.4561.

[14] E. A. Schauffert, G. Cusatis. Lattice discrete particle model for fiber-reinforced concrete. I: Theory. Journal of Engineering Mechanics 2011; 138.7: 826-833.

[15] R. Rezakhani, G. Cusatis, Asymptotic expansion homogenization of discrete finescale models with rotational degrees of freedom for the simulation of quasi-brittle materials. J. Mech. Phys. Solids 2016; 88: 320-345.

[16] R. Rezakhani and G. Cusatis, Generalized mathematical homogenization of the lattice discrete particle model, in Proceedings of the 8th International Conference on Fracture Mechanics of Concrete and Concrete Structures, FraMCoS 2013, pages 261-271, Toledo, Spain, 2013.

[17] S. Esna Ashari, G. Buscarnera, G. Cusatis, Micro-scale modeling of the inelastic response of a granular sandstone, Proceedings of 49th US Rock Mechanics/Geomechanics Symposium, San Francisco, California, US, June 2015.

[18] G. Cusatis. Strain-rate effects on concrete behavior. International Journal of Impact Engineering 2011; 38: 162-170. 